\title{
Población femenina de nacionalidad extranjera en la provincia de Barcelona, 1996
}

\author{
Andreu Domingo \\ Inés Brancós
}

Universitat Autònoma de Barcelona. Centre d'Estudis Demogràfics

08193 Bellaterra (Barcelona). Spain

adomingo@cedserver.uab.es

ibrancos@cedserver.uab.es

\section{Resumen}

Los datos que nos aporta la Estadística de Población anexa al Padrón de 1996 nos han permitido realizar una primera aproximación al perfil de la población femenina de nacionalidad extranjera residente en la provincia de Barcelona. Cuando nos referimos a este colectivo, parece que exista un estereotipo donde se refleja la imagen de una mujer dominicana o filipina. Las estadísticas, sin embargo, no se corresponden con nuestro prejuicio, ya que nos confirman que la nacionalidad con un mayor número de efectivos femeninos es la marroquí, por ello, el artículo ahonda en los factores que han intervenido en la creación de dicho estereotipo. Finalmente, y a partir del trabajo anterior, se reflexiona sobre posibles elementos que nos apunten tendencias futuras en relación con la presencia de población femenina de nacionalidad extranjera en la provincia de Barcelona.

Palabras clave: mujer, población extranjera, inmigración, distribución territorial, edad, nivel de instrucción, actividad, provincia de Barcelona.

\section{Abstract. Female foreign population in the province of Barcelona, 1996}

Population statistics data from the 1996 padrón (census) permitted us to make a first approximation of the profile of women living in the Province of Barcelona. When one refers to this group, it seems to exist a stereotype reflecting the image of a Philippine or Dominican woman. However, statistics do not conform to this stereotype, as the more numerous foreign nationality is the Moroccan one. This paper examines the factors that have contributed to the formation of this stereotype. We also consider elements that point out to future trends in relation to the presence of female foreign-nationals in the province of Barcelona.

Key words: woman, foreign population, immigration, geographic distribution, age, educational level, activity, province of Barcelona. 


\section{Sumario}

1. Introducción: $i$ Y las extranieras

2. ¿De qué nacionalidad es mi vecina?

La población femenina extranjera

por nacionalidades en la provincia de Barcelona

3. ¿Dónde reside? Distribución espacial de la población femenina con nacionalidad extranjera

4. ¿Cuántos años tiene? La composición por grupos de edad

5. ¿Qué estudios tiene? El nivel de instrucción de la población femenina de diez y más años
6. ¿A qué se dedican? La relación con la actividad

7. Imagen estadística de la población femenina de nacionalidad extranjera

8. La fuerza del estereotipo

9. Futuro: ¿feminización

de las migraciones internacionales?

Bibliografía

\section{Introducción: ¿Y las extranjeras?}

Cuando se habla de inmigración o de población extranjera, ¿cuál es la primera imagen que suele venirnos a la cabeza? Un hombre, joven y posiblemente marroquí. Esa imagen no parece adecuarse ni mucho menos al registro estadístico. En España, en 1997, según los datos publicados por la Comisión Interministerial de Extranjería del Ministerio del Interior, aunque la nacionalidad más representada sea la marroquí, casi la mitad de los 609.813 residentes de nacionalidad extranjera pertenecían a algún país europeo. ¿Y el sexo? Las 282.544 mujeres con permiso de residencia representaban el $46 \%$ del total, si además añadimos que existe un $4 \%$ de los permisos donde no consta el sexo, el porcentaje asciende a un $48 \%$. Ese porcentaje tampoco parece justificar nuestro prejuicio sobre el carácter preponderantemente masculino de la inmigración extranjera o sobre la población de nacionalidad extranjera. Si a nuestra imaginación le pedimos que se esfuerce por concentrarse en la nacionalidad de las inmigradas, tenemos muchas probabilidades de que la imagen que se nos dibuje sea la de mujeres filipinas o dominicanas, dedicadas al trabajo doméstico. Pues bien, una vez más el estereotipo parece alejarse a pasos agigantados del registro estadístico: en primer lugar nos encontramos con las 34.909 marroquíes, seguidas a corta distancia por las 34.526 británicas, representando cada una de ellas el $12 \%$ de las mujeres con permiso de residencia; en tercer lugar, constan las 24.731 alemanas, con un $9 \%$ del total. Muy por debajo aparecerán «nuestras» dominicanas y filipinas, con sendos 5,5 y 2,5\%.

$\mathrm{Si}$ exceptuamos las series concernientes a los permisos de trabajo en vigor, no será hasta el año 1997 en la que veamos publicada una mínima información por sexos, restringida a la nacionalidad de las residentes. De este modo, los primeros trabajos sobre la población extranjera femenina a partir de series estadísticas, se circunscribirán a las mujeres con permiso de trabajo en vigor según 
su nacionalidad (Varona y Daolio, 1994; Izquierdo, 1996). En dichas series estadísticas, efectivamente, el peso de las mujeres es menor, así, en 1997, de los 176.022 permisos vigentes en España, el 35\% correspondían a mujeres. Hasta ese momento, pues, era pertinente sostener un grave desconocimiento sobre las características de la población de nacionalidad extranjera por razón de sexo (Domingo, 1998).

En este artículo realizaremos una primera aproximación al perfil de la población femenina de nacionalidad extranjera en la provincia de Barcelona. Para ello analizaremos dicho colectivo mediante los datos del Padrón de 1996 en la provincia de Barcelona, recogidos por el Institut d'Estadística de Catalunya. Aunque censos y padrones presenten un mayor subregistro que las series estadísticas referidas a los residentes extranjeros elaboradas por la Dirección General de Policía, la estadística de población anexa al Padrón de 1996 es la fuente con más información sobre la población extranjera femenina de la que disponemos en la actualidad. Así, a parte de la nacionalidad, podemos obtener información sobre la edad, el nivel de instrucción para las mujeres de diez y más años, y la relación con la actividad. Es por esta razón que utilizaremos el Padrón de 1996 en nuestro análisis.

También es nuestro propósito explicar los elementos constructivos del estereotipo y ver cuan lejos o cuan cerca se sitúa éste de la realidad, o por lo menos de la imagen estadística. Por último, a partir del trabajo anterior, expondremos nuestras reflexiones sobre las tendencias futuras referentes a la presencia de población femenina de nacionalidad extranjera en la provincia de Barcelona.

\section{2. ¿De qué nacionalidad es mi vecina? La población femenina extranjera por nacionalidades en la provincia de Barcelona}

En el Padrón de 1996, en Cataluña se registraron 98.035 personas de nacionalidad extranjera, 51.485 eran hombres (53\%) y 46.550 mujeres (47\%), ello representa el 1,61\% de la población total empadronada en esta comunidad.

El porcentaje de población con nacionalidad extranjera respecto a la población total en la provincia de Barcelona se sitúa en el 1,54\%. De las 71.286 personas con nacionalidad extranjera registradas en esta provincia (el 72,7\% del total de Cataluña), 36.675 eran hombres (el 51\%) y 34.611 mujeres (el $49 \%$ ). Es decir, casi la mitad de la población de nacionalidad extranjera en la provincia de Barcelona era de sexo femenino.

Esa relación entre los sexos, resulta muy diferente, si lo que analizamos es el reparto por continentes: las 12.212 mujeres americanas que habitan en la provincia de Barcelona representan el 58,5\% de la población americana. En Europa y Oceanía la proporción es del 50\%, con 10.153 y 49 mujeres, respectivamente. Tan sólo encontramos una proporción inferior de mujeres entre la población asiática, donde las 3.456 mujeres representan el $47,5 \%$ y mucho menor en la africana, donde las 8.731 mujeres registradas alcanzan el 38\%.

El reparto continental entre la población extranjera femenina, como puede observarse en la tabla 1, es el siguiente: en primer lugar encontramos a las ame- 
Tabla 1. Población femenina de nacionalidad extranjera, por nacionalidades y grandes grupos de edad. Provincia de Barcelona, 1996.

\begin{tabular}{|c|c|c|c|c|c|}
\hline Nacionalidad & $0-14$ & $15-64$ & $>64$ & Total & $\%$ \\
\hline África & 2.167 & 6.341 & 223 & 8.731 & 25,23 \\
\hline Argelia & 25 & 84 & 8 & 117 & 0,34 \\
\hline Gambia & 109 & 270 & 0 & 379 & 1,1 \\
\hline Guinea Ecuatorial & 20 & 90 & 4 & 114 & 0,33 \\
\hline Marruecos & 1.915 & 5.331 & 187 & 7.433 & 21,48 \\
\hline Senegal & 9 & 93 & 2 & 104 & 0,3 \\
\hline Túnez & 2 & 2 & 0 & 4 & 0,01 \\
\hline Resto de África & 87 & 471 & 22 & 580 & 1,68 \\
\hline América & 1.253 & 9.838 & 1.121 & 12.212 & 35,28 \\
\hline Estados Unidos & 69 & 403 & 48 & 520 & 1,5 \\
\hline México & 32 & 236 & 20 & 288 & 0,83 \\
\hline Rep. Dominicana & 214 & 1.615 & 18 & 1.847 & 5,34 \\
\hline Honduras & 14 & 84 & 5 & 103 & 0,3 \\
\hline Cuba & 43 & 596 & 436 & 1.075 & 3,11 \\
\hline Costa Rica & 5 & 14 & 1 & 20 & 0,06 \\
\hline R. América N. y Central & 83 & 622 & 34 & 739 & 2,14 \\
\hline Argentina & 188 & 1.332 & 277 & 1.797 & 5,19 \\
\hline Chile & 77 & 601 & 57 & 735 & 2,12 \\
\hline Uruguay & 39 & 373 & 50 & 462 & 1,33 \\
\hline Brasil & 57 & 422 & 13 & 492 & 1,42 \\
\hline Venezuela & 48 & 325 & 33 & 406 & 1,17 \\
\hline Perú & 271 & 2.396 & 78 & 2.745 & 7,93 \\
\hline Colombia & 78 & 597 & 36 & 711 & 2,05 \\
\hline R. América del Sur & 35 & 222 & 15 & 272 & 0,79 \\
\hline Asia & 552 & 2.783 & 121 & 3.456 & 9,99 \\
\hline Filipinas & 141 & 1.284 & 39 & 1.464 & 4,23 \\
\hline China & 113 & 533 & 27 & 673 & 1,94 \\
\hline Japón & 37 & 197 & 4 & 238 & 0,69 \\
\hline India & 75 & 181 & 9 & 265 & 0,77 \\
\hline Resto de Asia & 186 & 588 & 42 & 816 & 2,36 \\
\hline Europa & 906 & 7.804 & 1.443 & 10.153 & 29,33 \\
\hline Alemania & 176 & 1.576 & 249 & 2.001 & 5,78 \\
\hline Bélgica & 28 & 211 & 34 & 273 & 0,79 \\
\hline Dinamarca & 4 & 79 & 8 & 91 & 0,26 \\
\hline Francia & 183 & 2.172 & 591 & 2.946 & 8,51 \\
\hline Grecia & 5 & 26 & 12 & 43 & 0,12 \\
\hline
\end{tabular}


Tabla 1 (continuación).

\begin{tabular}{lrrrrr}
\hline Nacionalidad & $\mathbf{0 - 1 4}$ & $\mathbf{1 5}-\mathbf{6 4}$ & $\boldsymbol{>} \mathbf{6 4}$ & \multicolumn{1}{c}{ Total } & $\%$ \\
\hline Europa & & & & & \\
Irlanda & 6 & 75 & 1 & 82 & 0,24 \\
Italia & 125 & 833 & 221 & 1.179 & 3,41 \\
Luxemburgo & 0 & 6 & 0 & 6 & 0,02 \\
Países Bajos & 46 & 392 & 37 & 475 & 1,37 \\
Portugal & 68 & 516 & 57 & 641 & 1,85 \\
Reino Unido & 102 & 942 & 83 & 1.127 & 3,26 \\
Áustria & 10 & 85 & 19 & 114 & 0,33 \\
Suecia & 13 & 88 & 10 & 111 & 0,32 \\
Noruega & 2 & 32 & 3 & 37 & 0,11 \\
Andorra & 20 & 83 & 14 & 117 & 0,34 \\
Suiza & 12 & 209 & 89 & 310 & 0,9 \\
Resto de Europa & 106 & 479 & 15 & 600 & 1,73 \\
\hline Oceanía & 5 & 43 & 1 & 49 & $\mathbf{0 1 1}$ \\
Australia & 5 & 32 & 1 & 38 & 0,11 \\
Resto de Oceanía & 0 & 11 & 0 & 11 & 0,03 \\
\hline Apátridas & $\mathbf{3}$ & 5 & $\mathbf{2}$ & $\mathbf{1 0}$ & $\mathbf{0 , 0 3}$ \\
\hline Total & $\mathbf{4 . 8 8 6}$ & $\mathbf{2 6 . 8 1 4}$ & $\mathbf{2 . 9 1 1}$ & $\mathbf{3 4 . 6 1 1}$ & $\mathbf{1 0 0 , 0 0}$ \\
\hline
\end{tabular}

Fuente: Estadística de Población, 1996 (IEC). Elaboración propia.

ricanas (un 35\%), en segundo lugar, a las europeas (29\%), seguidas de las africanas $(25 \%)$, de las asiáticas $(10 \%)$, y por último, las mujeres de Oceanía, cuyo porcentaje no llega al $1 \%$.

Aunque por continentes, como hemos visto, el número de americanas sea superior a cualquier otro contingente, por nacionalidades son las mujeres marroquíes, con mucho, las más numerosas. En efecto, las 7.433 mujeres marroquíes encabezan, a mucha distancia de las demás, la relación de las diez primeras nacionalidades con mayor número de mujeres residiendo en la provincia de Barcelona. En segundo lugar, tampoco veremos a las que el estereotipo nos tiene acostumbrados, son las 2.946 francesas las que ocupan esa posición, seguidas a corta distancia por las 2.745 peruanas. En cuarto lugar aparecen 2.001 mujeres de nacionalidad alemana, y no es hasta el quinto lugar de la clasificación que nos encontramos con las tan esperadas dominicanas, con $1.847 \mathrm{mu}-$ jeres. En sexto lugar las 1.797 argentinas. En séptimo, ¿por fin? a las 1.464 filipinas, seguidas de las italianas, británicas y cubanas, con efectivos alrededor de las 1.000 personas.

Parece que la imagen que por lo común se tiene de la inmigración femenina, hasta el momento no se corresponde, por lo menos en la provincia de Barcelona, con los datos disponibles: las mujeres son mucho más de las que 
se dicen, y entre éstas, la nacionalidad con más efectivos es la marroquí. Veamos a continuación como se distribuyen estas mujeres en el territorio.

\section{3. ¿Dónde reside? Distribución espacial de la población femenina con nacionalidad extranjera}

La primera característica que cabe resaltar en relación con la distribución territorial de las mujeres de nacionalidad extranjera sobre el territorio es su marcada concentración en un mismo municipio: Barcelona (mapa 1). Aunque también se da dicha concentración de la población masculina, entre las mujeres el porcentaje que reside en Barcelona es aún superior: las 15.285 mujeres con nacionalidad extranjera residentes en este municipio representan el $44 \%$ del total provincial, frente al $37,5 \%$ del total masculino.

Los demás municipios con más de 500 mujeres con nacionalidad extranjera se localizan, todos ellos, en la Región Metropolitana de Barcelona: 1.419 mujeres residen en Hospitalet de Llobregat, 886 en Terrassa, 862 en Castelldefels, 854 en Mataró, 834 en Badalona, 801 en Sant Cugat del Vallès y 667 en Sabadell. Entre estos municipios, la tipología por nacionalidades es bastante heterogénea. Así, la población africana, y en concreto, la marroquí, predomina en los siguientes municipios: Mataró, donde el $62 \%$ de las mujeres con nacionalidad extranjera son africanas; Terrassa, con el $45 \%$ de las mujeres, y Badalona, con el 39\%. A las mujeres africanas les siguen las del continente americano, siendo, entre ellas, las dominicanas las que cuentan con un porcentaje superior. En otro grupo de municipios destacan estadísticamente las mujeres americanas. Éstos son: Hospitalet de Llobregat, donde el 47,5\% de la población femenina con nacionalidad extranjera es americana; Barcelona, con el $42 \%$ y Sabadell, con el 46\%. En estos municipios predominan las dominicanas y las peruanas. Los municipios con más de 500 mujeres con nacionalidad extranjera y con predominio de europeas son: Sant Cugat del Vallès (el $53 \%$ de la población femenina considerada corresponde a esta tipología) y Castelldefels (con el 52\%). En los dos municipios, la nacionalidad que predomina es la alemana.

Resumiendo, los municipios con más número de habitantes, y en general aquellos situados en la región metropolitana de Barcelona son los que ejercen una mayor atracción sobre la población femenina extranjera. Dicho comportamiento es idéntico al observado históricamente en la dinámica migratoria en la provincia de Barcelona: siempre la inmigración femenina ha tenido un peso importante en dichos municipios. Ello es debido, sin lugar a dudas, a la ocupación de esas mujeres, que como veremos es preeminente en el sector servicios, cuya oferta se concentra en el territorio. De todos modos, podemos también observar que la representación de cada continente en cada municipio está estrechamente relacionada con las características de este municipio, en referencia al uso del territorio. Así, nos encontramos con una población femenina europea concentrada en los municipios claramente residenciales y turísticos de alto nivel, mientras que el grueso de las mujeres africanas residen 


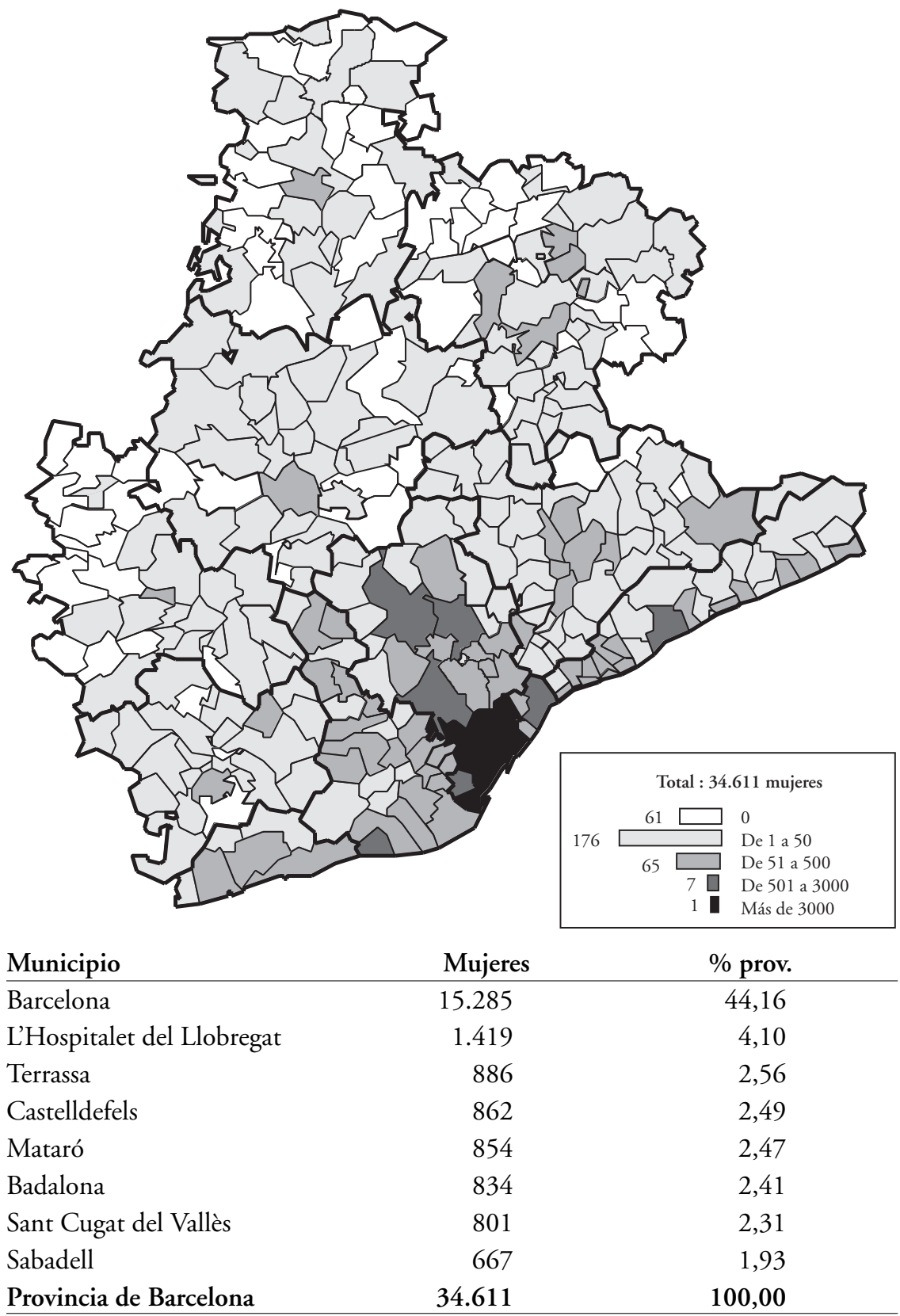

Fuente: Estadística de población, 1996 (IEC). Elaboración propia.

Mapa 1. Población femenina de nacionalidad extranjera, por municipios. Provincia de Barcelona, 1996. 
en municipios caracterizados bien sea por incluir una importante actividad económica, bien sea por ser municipios residenciales, aunque esta vez de nivel bajo. En cuanto a las mujeres americanas, siendo como son las mayoritarias, predominan en las ciudades residenciales. Por fin las mujeres asiáticas, las menos numerosas, son las que menos se reparten en el territorio.

\section{4. ¿Cuántos años tiene? La composición por grupos de edad}

Si la población femenina se distribuyera de forma uniforme sobre el territorio, habría muchas probabilidades de que nuestra vecina en 1996, además de ser marroquí, tuviera entre 30 y 34 años. La estructura por grandes grupos de edad de la población femenina de nacionalidad extranjera presenta sin embargo características muy diversas (tabla 1).

Entre los 0 y los 14 años encontramos 4.886 niñas que representan el 14\% de la población femenina de nacionalidad extranjera. Por continentes, su distribución es semejante a la de los 5.345 niños de nacionalidad extranjera en la provincia. Un $44 \%$ de esas niñas son africanas (2.167), un 26\% americanas (1.253), un $18,5 \%$ europeas (906) y un $11 \%$ asiáticas (552).

Entre los 15 y los 64 años hay 26.814 mujeres que suponen el $77 \%$ del total. La mayoría de las mujeres de este grupo de edad son, con un 37\%, americanas (9.838), les siguen las europeas con un 29\% (7.804), las africanas con un $24 \%(6.341)$ y con un $10 \%$, las asiáticas (2.783). A diferencia del anterior grupo de edad, la distribución continental de los 29.274 hombres es muy distinta: entre éstos, predomina la población africana, con un 39\% del total, seguida por los europeos con un $27 \%$, de los americanos con un $23 \%$ y finalmente de los asiáticos, con un $11 \%$.

Por último, hay 2.911 mujeres mayores de 64 años, que constituyen el 8\% de la población femenina, frente a 2.056 hombres, siendo éste el único grupo de edad donde predominan las mujeres. La mitad de la población anciana de nacionalidad extranjera y sexo femenino es europea (1.443), el 38\% americana (1.121), el $8 \%$ africana (223) y el $4 \%$ asiática (121). Estos porcentajes son parecidos entre los hombres, aunque el peso de los americanos sea ligeramente inferior, en beneficio de los restantes continentes.

Así pues, entre la población femenina, dependiendo del grupo de edad que observemos, la mayoría de cada uno pertenece a un continente distinto: población infantil eminentemente africana, joven-adulta americana y anciana europea.

La pirámide de edad de la población de nacionalidad extranjera (véase figura 1), tanto por el lado que representa los efectivos masculinos como por el que representa los efectivos femeninos, sobresale en las edades adultas, siendo, con todo, mucho más equilibrada la distribución de las mujeres. Mientras que en las mujeres el 50\% de la población está agrupada entre los 20 a 44 años, en los hombres esa misma proporción se consigue con los grupos entre 25 a 44 años. En ambos casos, el grupo 30-34 años es el más numeroso, abarcando en los hombres un $8 \%$ del total de la población de nacionalidad extranjera de la provincia y un $7 \%$ en las mujeres. 


\section{TOTAL}
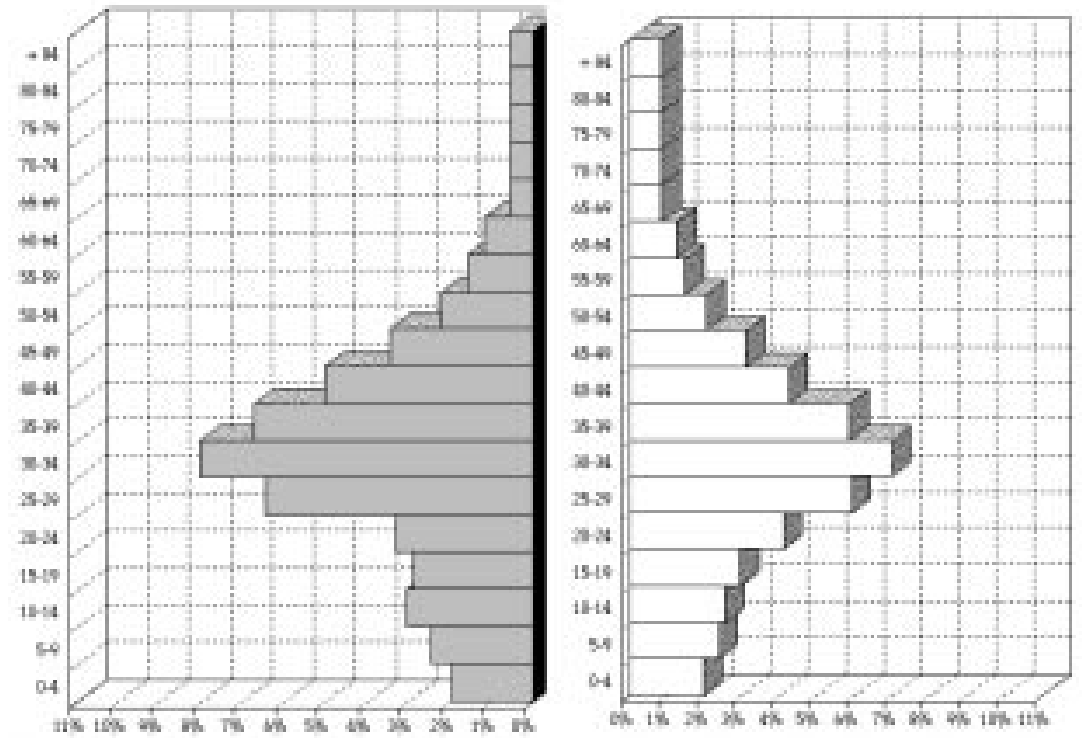

ÁFRICA

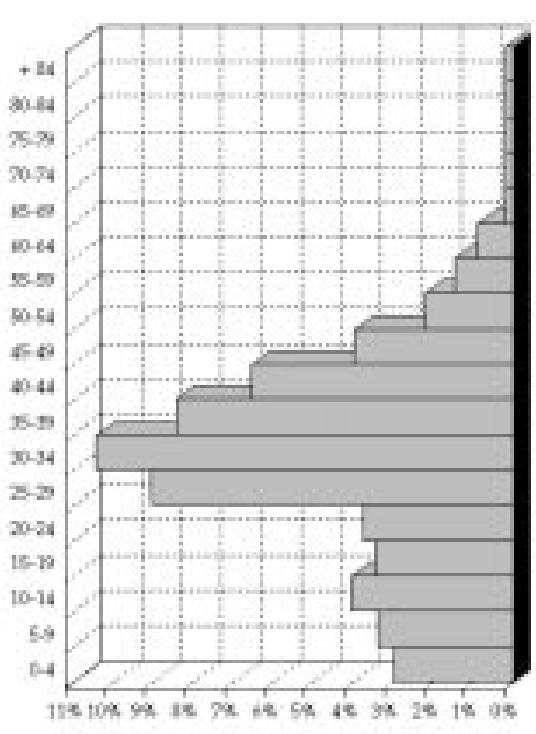

Hombres

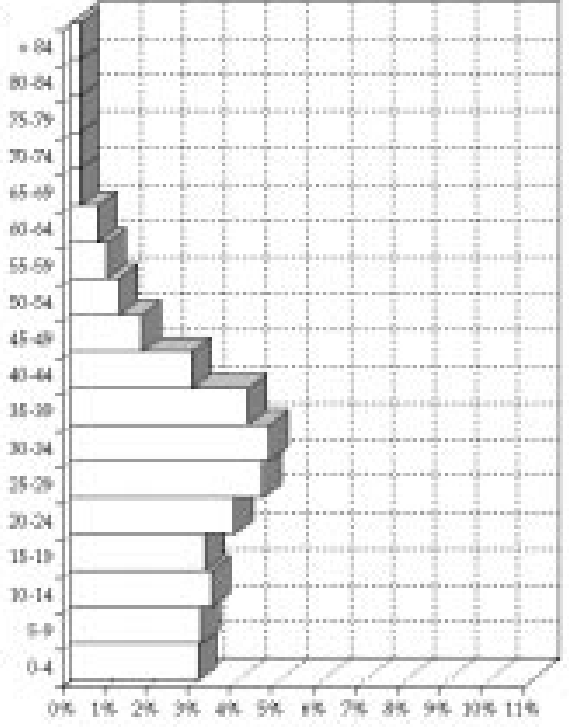

Mujeres

Figura 1. Pirámides de población de nacionalidad extranjera, por continentes. Provincia de Barcelona, 1996.

Fuente: elaboración propia. 


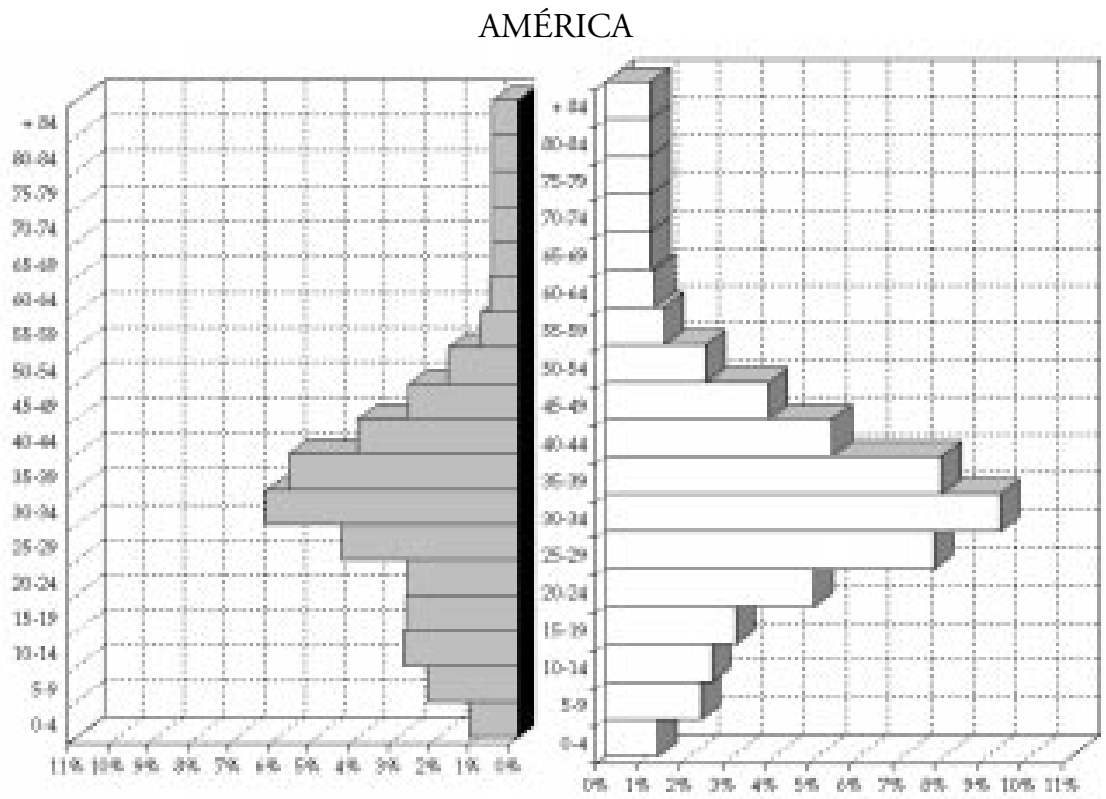

ASIA

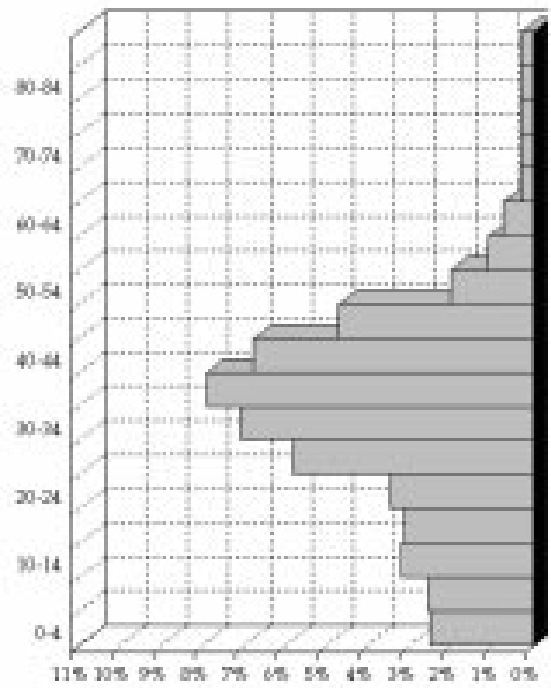

Hombres

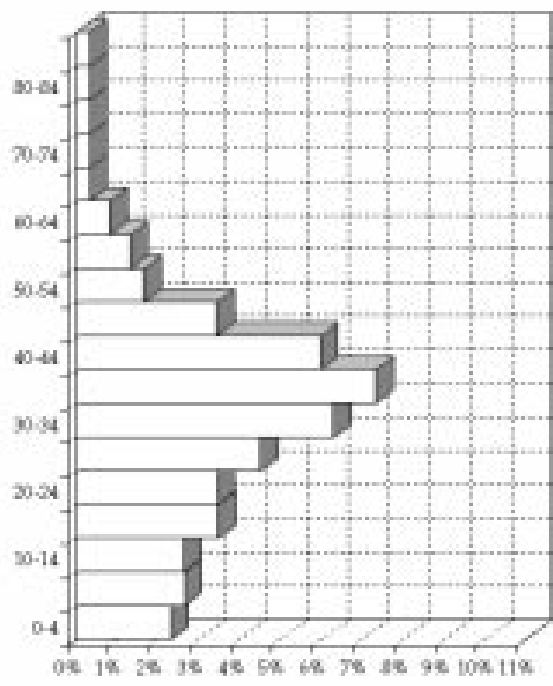

Mujeres

Figura 1. (Continuación). 


\section{EUROPA}

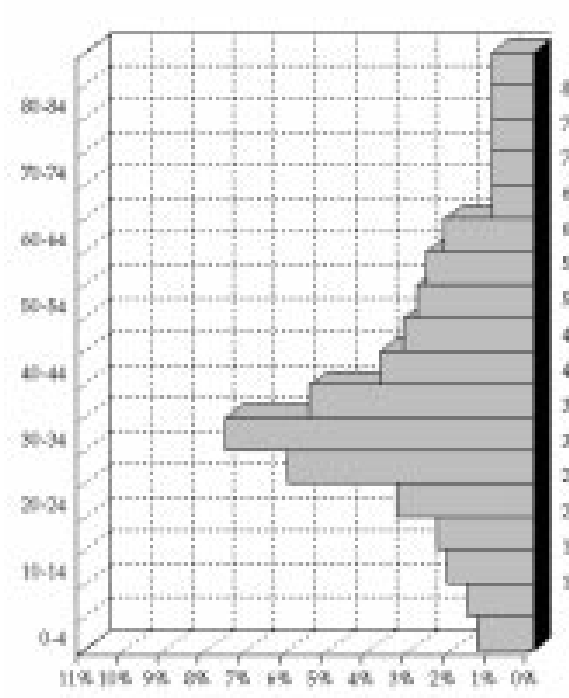

Hombres

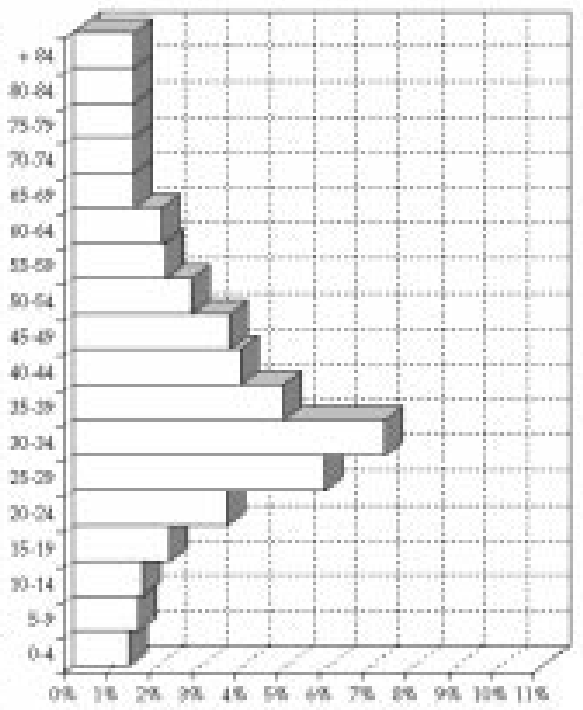

Mujeres

Figura 1. (Continuación).

Las pirámides de edad según los continentes, muestran todas una forma romboidal, es decir, con efectivos superiores en los grupos de 25 a 39 años, decrecientes en los extremos de las pirámides para ambos sexos. Teniendo en cuenta lo que se ha apuntado con anterioridad, en comparación al conjunto destaca, sin embargo, la presencia más notable de efectivos infantiles en la pirámide africana, contrastada con la menor de efectivos de ancianos, perfil inverso al observado en las pirámides europea y americana, donde hay una mayor presencia de efectivos a mayor edad, y menor en las primeras edades. El contraste más notable, sin embargo, lo obtenemos cuando contemplamos la relación entre los hombres y las mujeres en cada una de las pirámides. Si Europa y Asia muestran un perfil simétrico, África y América lo exhiben asimétrico, perceptiblemente favorable a uno de los dos sexos, en el caso africano, al masculino, mientras que en el caso americano, al femenino. No se trata tan sólo del número singularmente superior de efectivos masculinos en la pirámide africana, sino de su muy desigual repartición por edades, que hace que los hombres entre 30 y 34 años concentren el 10\% de la población total, mientras que los grupos de edades de las mujeres tan sólo incluyen el 5\%. Lo mismo, pero a la inversa, puede decirse de la pirámide americana, las mujeres entre 30 y 34 años representan el $9 \%$ del total, mientras que los hombres solamente reúnen al $6 \%$. 
Si lo que observamos es la desagregación por grupos quinquenales, veremos que, a excepción de la población asiática, el grupo más señalado es el que tiene entre 30 y 34 años, como ya habíamos mencionado, con porcentajes que oscilan entre el $12 \%$ para este grupo de edad entre las africanas al $16 \%$ que ostenta entre las americanas. Entre las mujeres asiáticas el grupo más representado es el correspondiente a los 35-39 años.

La estructura de edad constituirá una de las determinantes de los diferentes niveles de instrucción y de la relación con la actividad registradas entre la población femenina por continentes y nacionalidades, veamos a continuación esas dos características de la población femenina inmigrada.

\section{5. ¿Qué estudios tiene? El nivel de instrucción de la población femenina de diez y más años}

El porcentaje que las mujeres detentan en cada uno de los distintos niveles de instrucción no presenta grandes diferencias con el de los hombres a esos mismos niveles. De hecho, las mujeres tienen proporciones ligeramente más elevadas en los niveles equivalentes a la segunda etapa de EGB, al primer grado de FP, al BUP y COU, y al título medio, entre un 52 y un $53 \%$, siendo en cambio menos representadas en la primaria incompleta y en el extremo opuesto, en el título superior, con un $43 \%$.

Entre las 31.535 mujeres de nacionalidad extranjera de diez y más años, como puede calcularse a partir de la tabla 2 , el nivel de instrucción con el porcentaje más elevado, al igual que entre los hombres, es el equivalente a la primera etapa de EGB, un $23 \%$ del total (7.125 mujeres y 7.627 hombres). En segundo lugar, para las mujeres encontramos el nivel correspondiente al BUP y COU con el $18 \%$ (5.533 mujeres) y en tercero, la primaria incompleta con el 14\% (4.369). Entre los hombres ese orden se invierte, siendo la primaria incompleta la que ocupa el segundo lugar con el 17\%, y BUP y COU el tercero, con el $15 \%$ de la población masculina.

El 81\% de las 794 mujeres que no saben leer ni escribir y el 58\% de las 4.369 mujeres con la primaria incompleta son africanas. En cambio, en los niveles de primera y segunda etapa de EGB, aparecen con más frecuencia las americanas con el 36 y el 41\%. También son las mujeres del continente americano las que ostentan unos porcentajes más elevados en primer grado de FP con el $39 \%$ y en BUP y COU con el $43 \%$. Las europeas son mayoría entre las mujeres con el segundo grado de FP con un $41 \%$, con el título medio y con un título superior con el 43 y el $44 \%$ respectivamente.

Por continentes, del mismo modo que la estructura por edades implicaba diferencias significativas, el nivel de instrucción muestra notables disparidades. De esta forma, el más representado entre las 7.329 mujeres africanas es la primaria incompleta con el 35\% (2.540 mujeres), en segundo lugar la primera etapa de EGB con el 30\% (2.186 mujeres) y en tercer lugar la segunda etapa de EGB, con el 11\% (822 mujeres). De las 11.482 americanas el 22\% han cursado hasta la primera etapa de EGB (2.550), seguidas de las que tienen 
Tabla 2. Población femenina de nacionalidad extranjera de diez y más años, por continentes, según nivel de instrucción. Provincia de Barcelona, 1996.

\begin{tabular}{|c|c|c|c|c|c|c|c|c|c|c|}
\hline Nacionalidad & $\begin{array}{l}\text { No leer } \\
\text { o escribir }\end{array}$ & $\begin{array}{l}\text { Primaria } \\
\text { incompleta }\end{array}$ & $\begin{array}{l}\text { EGB } \\
1^{a} \text { etapa }\end{array}$ & $\begin{array}{l}\text { EGB } \\
2^{a} \text { etapa }\end{array}$ & $\begin{array}{l}\text { FP } \\
1^{\circ} \text { grado }\end{array}$ & $\begin{array}{l}\text { FP } \\
2^{\circ} \text { grado }\end{array}$ & $\begin{array}{l}\text { BUP } \\
\text { y COU }\end{array}$ & $\begin{array}{l}\text { Título } \\
\text { medio }\end{array}$ & $\begin{array}{l}\text { Título } \\
\text { superior }\end{array}$ & Total \\
\hline África & 646 & 2.540 & 2.186 & 822 & 232 & 119 & 434 & 181 & 169 & 7.329 \\
\hline América & 44 & 858 & 2.550 & 1.742 & 564 & 484 & 2.405 & 1.298 & 1.537 & 11.482 \\
\hline Asia & 37 & 318 & 737 & 432 & 182 & 98 & 614 & 367 & 310 & 3.095 \\
\hline Europa & 67 & 649 & 1.644 & 1.199 & 470 & 492 & 2.067 & 1.416 & 1.571 & 9.575 \\
\hline Oceanía & 0 & 2 & 5 & 5 & 2 & 2 & 12 & 4 & 14 & 46 \\
\hline Apátridas & 0 & 2 & 3 & 0 & 2 & 0 & 1 & 0 & 0 & 8 \\
\hline Total & 794 & 4.369 & 7.125 & 4.200 & 1.452 & 1.195 & 5.533 & 3.266 & 3.601 & 31.535 \\
\hline
\end{tabular}

Fuente: Estadística de Població, 1996 (IEC). Elaboración propia. 
BUP y COU con el 21\% (2.405) y en tercer lugar las que han finalizado la segunda etapa de EGB con el 15\% (1.742). El nivel de instrucción entre las 3.095 mujeres asiáticas revela una distribución similar a la observada entre las americanas: en primer lugar encontramos la primera etapa de EGB, con el $24 \%$ (737), en el segundo, BUP y COU, con el 20\% (614) y, en tercer lugar, el $14 \%$ que han cursado la segunda etapa de EGB (432). En el $22 \%$ de las mujeres europeas su nivel de instrucción más elevado es el de BUP y COU (2.067). En segundo lugar encontramos un 17\% (1.644) correspondiente a la segunda etapa de EGB y en tercer lugar, con un 16\% (1.571), las mujeres con titulación superior.

Por nacionalidades encontramos casos atípicos en comparación con el conjunto continental. Por ejemplo, dentro del continente africano destacaremos las mujeres ecuatoguineanas por el elevado porcentaje de titulación superior, excepcional dentro del conjunto continental: las 16 mujeres con nacionalidad de Guinea Ecuatorial y titulación superior representan el 16\% del total. En el polo opuesto encontramos a las 58 mujeres gambianas que no saben leer ni escribir, que suponen el $20 \%$ de la población femenina de Gambia. Del mismo modo, un 5\% de las 598 mujeres portuguesas mayores de 9 años declaran no saber leer ni escribir, porcentaje altísimo teniendo en cuenta que para el conjunto europeo es del 0,7\%.

El nivel de instrucción alcanzado no siempre se corresponde con la actividad que se realiza, sobre todo entre la población inmigrada de nacionalidad extranjera. Veamos cual es la relación con la actividad de estas mismas mujeres.

\section{6. ¿A qué se dedican? La relación con la actividad}

Si lo que analizamos es la relación que mantienen las 34.611 mujeres con nacionalidad extranjera residentes en 1996 en la provincia de Barcelona, con la actividad económica en la tabla 3 se muestra que más de una cuarta parte de ellas, 10.129 mujeres, se encuentran ocupadas. Un número parecido, $9.100 \mathrm{mu}-$ jeres, se declaran amas de casa, ocupando el tercer lugar las 6.409 estudiantes que vienen a representar el $18 \%$ del total de mujeres.

Exceptuando África, estos datos globales mantienen unos patrones similares por continentes. La principal relación con la actividad es la ocupada, con porcentajes que rondan el 30\% (4.098 americanas, 1.127 asiáticas, 3.327 europeas y 22 procedentes de Oceanía). En segundo lugar aparecen las amas de casa, con casi una cuarta parte del total de cada continente $(2.854$ americanas, 833 asiáticas, 2.421 europeas y 8 de Oceanía), seguidas de las estudiantes, con porcentajes que van del $12 \%$ de Oceanía al $21 \%$ de las mujeres con nacionalidad asiática (2.099 americanas, 719 asiáticas, 1.546 europeas y 6 de Oceanía). Como ya se ha mencionado, la distribución de las mujeres africanas en relación con la actividad que desarrollan y/o declaran, presenta un perfil distinto a las anteriores: predominio de las amas de casa con un 34\% del total de ellas (2.980); en segundo lugar y con un $23 \%$ se encuentran las estudiantes (2.035), y, ya en tercer lugar, aparecen las ocupadas con un $18 \%$ (1.554 mujeres). 
Tabla 3. Población femenina de nacionalidad extranjera, por continentes, según relación con la actividad económica. Provincia de Barcelona, 1996.

\begin{tabular}{|c|c|c|c|c|c|c|c|c|c|}
\hline Nacionalidad & Ocupada & $\begin{array}{l}\text { Desocupada } \\
\text { primer } \\
\text { trabajo }\end{array}$ & $\begin{array}{l}\text { Desocupada } \\
\text { con trabajo } \\
\text { anterior }\end{array}$ & Jubilada & $\begin{array}{l}\text { Incapacitada } \\
\text { para } \\
\text { trabajar }\end{array}$ & Estudiantes & $\begin{array}{l}\text { Trabajos } \\
\text { hogar }\end{array}$ & $\begin{array}{l}\text { Otras } \\
\text { situaciones }\end{array}$ & Total \\
\hline África & 1.554 & 499 & 602 & 253 & 50 & 2.035 & 2.980 & 758 & 8731 \\
\hline América & 4.098 & 477 & 1.184 & 990 & 60 & 2.099 & 2.854 & 450 & 12.212 \\
\hline Asia & 1.127 & 110 & 256 & 154 & 42 & 719 & 833 & 215 & 3.456 \\
\hline Europa & 3.327 & 210 & 825 & 1.395 & 55 & 1.546 & 2.421 & 374 & 10.153 \\
\hline Oceanía & 22 & 0 & 4 & 3 & 1 & 6 & 8 & 5 & 49 \\
\hline Apátridas & 1 & 0 & 0 & 1 & 0 & 4 & 4 & 0 & 10 \\
\hline Total & 10.129 & 1.296 & 2.871 & 2.796 & 208 & 6.409 & 9.100 & 1.802 & 34.611 \\
\hline
\end{tabular}

Fuente: Estadística de Població, 1996 (IEC). Elaboración propia. 
A diferencia de lo que sucede con el análisis de la relación con la actividad masculina por nacionalidades donde, en su mayoría, se encuentran ocupados, entre las féminas y, dependiendo de la nacionalidad, predominan las ocupadas o bien las amas de casa. En términos generales, entre las americanas y las europeas el porcentaje de ocupadas es superior al resto de actividades, mientras que entre las africanas se encuentra un claro predominio de las amas de casa. A pesar de ello, existen claras excepciones. Por citar unos ejemplos: entre las mujeres ecuatoguineanas, el $32 \%$ son estudiantes y la mayoría de las mujeres cubanas (34\%) se alejan aún más del patrón mencionado, siendo éstas jubiladas.

Debido al volumen que representan sobre el total de mujeres, las de nacionalidad marroquí (7.433 mujeres), francesa (2.946) y peruana (2.745), -las tres principales nacionalidades de las mujeres residentes en la provincia de Barcelona-, son también las que presentan más ocupadas (1.295 mujeres marroquíes, 1.274 peruanas y 949 francesas), de desocupadas, habiendo trabajado con anterioridad (494 mujeres marroquíes, 319 peruanas y 279 francesas) y de las que se declaran amas de casa (2.545 mujeres marroquíes, seguidas de las 627 francesas y de las 478 peruanas).

Este esquema se rompe si lo que analizamos es la relación de las mujeres con las demás actividades: las marroquíes (416), las dominicanas (101) y las peruanas (80) son las principales nacionalidades entre las mujeres que aspiran a un primer trabajo; las nacionalidades que predominan entre las mujeres jubiladas se alejan, así mismo, del estereotipo que posiblemente pudiésemos tener en mente, el de la población europea. Si bien el volumen de jubiladas francesas es sensiblemente superior a las demás (610 que representan más de una cuarta parte del total de las jubiladas), a ellas les siguen las cubanas (con 371) y las argentinas (con 242 mujeres); el 28\% de las estudiantes tienen nacionalidad marroquí (1.783 mujeres), seguidas a mucha distancia estadística de las peruanas (428), las argentinas y las alemanas (345 y 344 respectivamente).

Una vez más nuestro estereotipo parece no ajustarse con la imágen estadística. Aún cuando es cierto que la mayoría de mujeres ocupadas son americanas, por nacionalidades vuelven a ser la mujeres marroquíes las que cuentan con más efectivos, seguidas de las peruanas y de las francesas. A continuación presentaremos un sucinto repaso a la imagen de la mujer de nacionalidad extranjera que se desprende del análisis del Padrón de 1996, para poder entender en el siguiente apartado cual es la distancia que separa esa imagen de la que nos impone el estereotipo.

\section{Imagen estadística de la población femenina de nacionalidad extranjera}

Como hemos visto, las 34.611 mujeres representan casi la mitad de la población de nacionalidad extranjera registrada en el Padrón de 1996, para la provincia de Barcelona, exactamente el $49 \%$. El 35\% de esas mujeres posee nacionalidad de algún país del continente americano (12.212), el 30\% son europeas (10.153), el 25\% son africanas (8.731), el 10\%, asiáticas (3.456), 
y con menos del 1\%, de Oceania (49). Por nacionalidades las 7.433 mujeres marroquíes son las más numerosas, seguidas de las 2.946 francesas y de las 2.745 peruanas.

La población femenina de nacionalidad extranjera, al igual que la masculina, reside preferentemente en la ciudad de Barcelona (el 44\%) y en la región metropolitana de Barcelona.

Su estructura por edades refleja fuertes desigualdades atendiendo a su origen continental: las 26.814 jóvenes adultas que constituyen el $77 \%$ del total son en su mayor parte americanas (el 37\%); las 4.886 menores de quince años con un $14 \%$ del total son predominantemente africanas (44\%), y las 2.911 ancianas, que representan el $8 \%$ del total, cuentan con una mayoría de europeas (50\%).

La primera etapa de EGB es el nivel de estudios realizado con un porcentaje más elevado entre las 31.535 mujeres de diez y más años, representando el $23 \%$ (con 7.125 mujeres). Le sigue el nivel de BUP y COU, con un $18 \%$ (5.533), y en tercer lugar, la primaria incompleta con un $14 \%(4.369)$.

La principal relación con la actividad es la de ocupada, con el $29 \%$ del total (10.129), en segundo lugar las amas de casa con el 26\% (9.100) y en tercer lugar, las estudiantes con un 18,5\% (6.409 mujeres).

¿A qué se debe que la imagen, aunque forzosamente esquemática, que nos muestran las estadísticas no se corresponda con nuestro prejuicio? A despecho de su importancia numérica, las mujeres siguen siendo invisibles en nuestro estereotipo. Como señala Dolores Juliano (1994), las fronteras de género contribuyen a eclipsar su presencia. ¿Qué factores concretos han intervenido en la creación del estereotipo sobre la inmigración y la población femenina de nacionalidad extranjera en España? ¿Cuál es la fuerza que sigue manteniéndolo?

\section{La fuerza del estereotipo}

En primer lugar, deberemos suponer que cuando se habla de población inmigrada o de población de nacionalidad extranjera, independientemente de su sexo, se está pensando en migraciones de trabajadores procedentes de países en vías de desarrollo. Ese primer sesgo interesado explica que no se suela considerar a la inmigración europea, por lo menos a la originaria de la Europa Occidental, cuando se habla de población de nacionalidad extranjera.

¿Por qué las dominicanas o las filipinas parecen acaparar nuestra imagen de la mujer inmigrada? Varios factores han determinado la fijación del estereotipo en esas dos nacionalidades:

Primero, es cierto que entre la población inmigrada de nacionalidad filipina o dominicana son, por lo general, mujeres (el 61 y el $77,5 \%$ respectivamente). El hecho de que las mujeres marroquíes, muchísimo más numerosas, no aparezcan (7.433 marroquíes frente a 1.847 dominicanas o 1.464 filipinas), podría explicarse teniendo en cuenta que quedan ensombrecidas por la elevada proporción de hombres marroquíes (las mujeres son el 38\% del total de la población marroquí en la provincia de Barcelona). 
En segundo lugar, deberemos considerar que tanto dominicanas como filipinas cumplen el requisito de encontrarse mayoritariamente ocupadas (el $38 \%$ entre las dominicanas, el $40 \%$ entre las filipinas), y que ese nivel de ocupación se ve además corroborado por las solicitudes de trabajo generalmente femeninas entre ambas nacionalidades (en 1997 el 87\% de las solicitudes de trabajo presentadas por dominicanos, y el $64 \%$ de los filipinos corresponden a mujeres).

En tercer lugar, ambas nacionalidades se dedican, dentro del sector servicios, al trabajo doméstico asalariado, por esa misma razón las mujeres marroquíes vuelven a ser injustamente olvidadas, el hecho de que buena parte de ellas se dediquen al trabajo doméstico no asalariado (el 34\%), las hace invisibles a un estereotipo fijado sobre la participación en el mercado de trabajo, aún cuando el número de mujeres marroquíes ocupadas (1.295) tampoco sea desdeñable, sin contar con aquellas dedicadas al trabajo informal.

A ello deberemos añadir factores históricos en los que se produce el movimiento migratorio: la inmigración filipina y dominicana se dio en el tiempo con anterioridad al incremento de la inmigración peruana. De este modo, esa anterioridad fue formativa y fijó el estereotipo, a despecho de que hoy en día residan más mujeres peruanas en la provincia de Barcelona que filipinas o dominicanas (2.745 peruanas), o que las 1.666 solicitudes de permiso de trabajo efectuadas por peruanas en 1997 representen casi un cuarto del total de las solicitudes femeninas. También, por desgracia, deberemos recordar el impacto que tuvo sobre la sociedad española el asesinato en Aravaca de Lucrecia Pérez, joven inmigrada dominicana en diciembre de 1992, un año después de la primera regularización extraordinaria, y el efecto magnificador que significó su repercusión debido a los medios de comunicación. La toma de conciencia de la xenofobia en España como realidad ligada a la inmigración extranjera quedó indeleblemente marcada por este acontecimiento.

Por último, pero no por ello menos determinante, la complementariedad simbólica en los orígenes, asiático y americano, se refuerza en el caso dominicano por la complementariedad también simbólica con la que se ha percibido la inmigración dominicana, desproporcionadamente difundida por los medios de comunicación: las mujeres dominicanas, se creía, o se dedicaban al trabajo doméstico o eran víctimas de las redes de traficantes de inmigradas. El valor simbólico atribuido oscila entre la percepción del trabajo asalariado de la mujer como una simple extensión del trabajo doméstico o, en el caso de la prostitución, como el paradigma del cuerpo femenino como objeto de consumo.

El prejuicio, visto de este modo, explica los factores que han intervenido en la construcción social de nuestra imagen de la mujer de nacionalidad extranjera. Que tal equívoco perdure en el futuro, depende de la información estadística que recibamos, y que nos permita acercarnos más a sus características sociodemográficas. Pero, llegados a este punto, es inevitable que nos preguntemos sobre su futuro. 


\section{Futuro: ¿¿feminización de las migraciones internacionales?}

Anticipamos nuestra hipótesis: creemos que en un futuro la presencia de población femenina de nacionalidad extranjera será más perceptible y se incrementará. En el contexto internacional, reputadas autoras como Lin Lean Lim (1997) sostienen el futuro aumento de la movilidad femenina como consecuencia del proceso de globalización y las tendencias que apuntan hacia la flexibilización del mercado laboral, el caso español no será una excepción, bien al contrario puede constituir un ejemplo paradigmático. Pero antes de exponer las razones por las cuales estamos convencidos del incremento de mujeres, tanto en números absolutos como en términos relativos, frente a los hombres de nacionalidad extranjera, queremos hacer mención a dos cuestiones previas, a nuestro juicio fundamentales.

Parte de ese supuesto incremento puede ser debido a la invisibilidad estadística de las mujeres en el pasado. Si no es hasta 1997 que podemos discriminar por razón de sexo las series estadísticas de residentes extranjeros, tampoco podemos asegurar con certeza cual era su presencia anteriormente. Algunas autoras como Hania Zlotnik (1998) han sostenido dicha hipótesis para el marco internacional.

En segundo lugar, hay que referirse a los elementos conceptuales que definen nuestras poblaciones, la definición de población extranjera es una definición legal. Parece obvio recordarlo, pero es necesario, su mayor o menor presencia pues, con independencia de los flujos migratorios que pueden alimentar esa población, depende de la definición y aplicación de la legislación en materia de nacionalización. Dicho de otro modo, si como es sabido ciertas nacionalidades acceden con más facilidad a la nacionalidad, en este caso española, o si, lo que queda por demostrar, la nacionalización por razón de sexo se aplicara con intensidad diferente para hombres o mujeres, el resultado sería que la presencia en las series estadísticas futuras de ciertas nacionalidades y de uno de los sexos sería mayor o menor. Parece evidente que si la legislación española favorece la nacionalización de ciertos países latinoamericanos por encima del resto (por poner un ejemplo), y sabiendo que entre éstos son mayoritarias las mujeres, su número se vería sujeto a mayores fluctuaciones. En definitiva, no perdamos de vista como predefinimos nuestra población objeto de estudio.

Una vez alertados sobre esas dos consideraciones previas, estadística y legal, como adelantábamos, varios elementos nos hacen creer que la presencia de población femenina de nacionalidad extranjera será creciente, en comparación con los hombres. En primer lugar apuntaremos que según el Anuario Estadístico de Extranjería del Ministerio del Interior, desde 1993 los permisos de trabajo en vigor, tanto para hombres como para mujeres, han ido en aumento, una vez superado el decrecimiento posolímpico, que el mismo año 1993 representó en la provincia de Barcelona siendo, no obstante, ese crecimiento mucho más pronunciado en el caso de los permisos otorgados a mujeres. Así, si en 1991 el número de permisos para los hombres era de 18.135 , y para las mujeres era de 6.969, en el año 1997 los hombres eran 24.892 mientras que las 
mujeres, 14.207. Durante este período, las mujeres han más que doblado el número de permisos, pasando de representar en 1991 el 28\% del total de permisos al 36\% en 1997. Si además tenemos en cuenta que el sector servicios es el que más ha crecido durante todos estos años, que en éste es donde se encuentran ocupadas mayoritariamente las mujeres (en 1997 trabajaban en dicho sector el $64 \%$ del total) y que en las solicitudes de permisos presentadas durante este año para el sector servicios, las mujeres rebasan a los hombres $(3.373 \mathrm{hom}$ bres y 3.840 mujeres), podemos sostener la hipótesis de un futuro crecimiento sostenido del trabajo asalariado de las mujeres de nacionalidad extranjera. Por otra parte, la incorporación de la mujer española en el mercado de trabajo alimentará la demanda de empleo en el sector doméstico, a cubrir previsiblemente por mujeres de nacionalidad extranjera. A ello deberíamos añadir, como señala Adriana Kaplan (1996), la decisión de favorecer la inmigración de las mujeres "productoras» sobre las "reproductoras», reflejado en la política de cupos que desde 1994 se viene aplicando en España y la dificultad creciente de la reagrupación familiar, asumiendo que las llamadas productoras constreñidas a la esfera doméstica ajena, carecerán de tiempo y condiciones para reproducirse.

Del mismo modo, deberemos entender que si se incrementa el número de personas jubiladas que eligen la provincia de Barcelona para residir, eso significaría un incremento en el número de mujeres, siendo como son las mujeres jubiladas mayoría (debido a la superior longevidad de las mujeres), y ello representaría el 52\% del total de la población jubilada.

A un futuro incremento de la inmigración femenina con intención de desempeñar una actividad económica como ocupadas o por causa de su jubilación, deberemos añadir un hipotético crecimiento del número de inmigradas por razón de estudios. Según el Anuario Estadístico de Extranjería del Ministerio del Interior, de los 3.386 estudiantes extranjeros con el permiso relativo en 1997 , el $53 \%$ eran mujeres. De todos es sabido que la entrada en la universidad de las generaciones vacías correspondientes a los españoles y españolas nacidos desde 1975, está creando una importante demanda potencial de estudiantes extranjeros a esos niveles, ya visible a nivel de tercer ciclo. Demanda que no parece arriesgado sostener que encuentre un mercado idóneo entre la población estudiante latinoamericana.

Por último, y aquí sí, sin que podamos demostrarlo cuantitativamente por falta de datos, no podemos sustraernos a la consideración de dos elementos que creemos que también pueden influir en inclinar la balanza a favor de la feminización de la población de nacionalidad extranjera en un futuro próximo: la reagrupación familiar y el mercado matrimonial.

Aunque no disponemos de datos desagregados por sexo relativos a la reagrupación familiar, sí que podemos aventurar que se reagrupan en números absolutos más mujeres que hombres, aunque tan solo sea porque la nacionalidad que más reagrupa, la más numerosa, la marroquí, es mayoritariamente masculina, y debemos suponer que la reagrupación afecta principalmente a las cónyuges de dichos inmigrados. Entre las nacionalidades donde la población 
femenina es más numerosa, en cambio, sabemos que parte de esa reagrupación se da con familiares femeninos, por razones de trabajo, hermanas, hijas o madres, con tanta intensidad, si no mayor que en el caso de los cónyuges; de todos modos, sea tenido este enunciado como hipotético, a falta de datos concluyentes.

Nuestra segunda especulación sigue las hipótesis esbozadas por la demógrafa Anna Cabré (1994). Si, como ella sostiene y nosotros compartimos, el efecto de la baja fecundidad que España ha experimentado en el último cuarto de siglo va a reflejarse en el mercado matrimonial provocando una escasez relativa de mujeres, aunque de forma minoritaria, el abastecimiento de esa demanda se hará a través de la inmigración internacional. Los pocos datos publicados sobre matrimonios mixtos nos informan que, por ejemplo, de los 9.067 matrimonios entre ciudadanos de diferentes nacionalidades contraídos en España en 1995, 4.532 tuvieron un cónyuge español y una cónyuge de nacionalidad extranjera, mientras que fueron 3.514 los matrimonios donde la contrayente era de nacionalidad española y el contrayente de nacionalidad extranjera. No se trata tan sólo de esa mínima diferencia, eso sí repetida año tras año (por lo que se puede observar en series anteriores) favorable a las mujeres de nacionalidad extranjera, sino que examinando las nacionalidades, parece razonable suponer que en el caso de esas mujeres no españolas, sea el matrimonio precisamente la razón de la inmigración para algunas nacionalidades muy concretas, mientras que en el de los hombres esa sospecha se reduce de forma ostensible.

Por todo ello creemos que la mujer inmigrada cada vez será más visible tanto social como estadísticamente. Socialmente, a consecuencia de su mayor presencia en la esfera pública y al lugar central que la mujer ocupa como reproductora social y biológica. Debe considerarse también su mayor visibilidad estadística, ya sea por la ya mencionada intensificación del flujo migratorio femenino, así como por una más amplia transferencia de información de dicho colectivo.

\section{Bibliografía}

Brancós, Inés; Domingo, Andreu (1997). «Aspectes demogràfics». En II informe sobre immigració i treball social. Barcelona: Diputació de Barcelona, Servei de Serveis Socials, p. 161-209.

CABRÉ, Anna (1994). «Tensiones inminentes en los mercados matrimoniales». En NADAL, J. El mundo que viene. Madrid: Alianza Editorial, p. 37-62.

Domingo, Andreu (1998). «La mujer inmigrada tras el velo estadístico». Papers de Demografia, núm. 146.

- (1999). «Visibilitat estadística i població estrangera». En Dones i migració a la Mediterrània Occidental. Barcelona: Institut de la Dona; Institut Català de la Mediterrània; Proa, p. 249-260.

IEC (1996). Estadistica de Població. Barcelona: Institut d'Estadística de Catalunya. IZQUIERDO, Antonio (1996). "La situación de la mujer inmigrante en España». En IZQUIERDO, A. (1996) La inmigración inesperada. La población extranjera en España (1991-1995). Madrid: Editorial Trotta, p. 117-125. 
JulianO, Dolores (1996). «Las mujeres inmigrantes, un plus de extranjeridad». En KAPLAN, Adriana (coord.). Procesos migratorios y relaciones interétnicas. VII Simposio del VII Congreso de Antropología y Sociología, Zaragoza, 1996. Zaragoza: Instituto Aragonés de Antropología y Federación de Asociaciones de Antropología del Estado Español, p. 61-70.

KAPLAN MARCUSÁN, Adriana (1996). «Introducción». En KaPLAN, Adriana (coord.). Procesos migratorios y relaciones interétnicas. VII Simposio del VII Congreso de Antropología y Sociología, Zaragoza, 1996. Zaragoza: Instituto Aragonés de Antropología y Federación de Asociaciones de Antropología del Estado Español, p. 9-12.

LEAM LIM, Lin (1997). "Flexible Labour Markets in a Globalizing World: The Implications for International Female Migration». En IUSSP; COMITTE ON SOUTHNorth Migration y Instituto Universitario OrTega y Gasset (1997). International Migration at Century's End: Trends and Issues. Barcelona, Spain, May 7-10, 1997. Liège: IUSSP, p. 1-24.

VARONA, Mary; DaOLIO, Nani (1994). Inmigración en España: femenino y plural. Madrid: Federación de Mujeres Progresistas.

ZlotNiK, Hania (1998). «La migración de mujeres del Sur al Norte». En MALGESINI, Graciela (comp.). Cruzando fronteras. Migraciones en el sistema mundial. Barcelona: Icaria/Fundación Hogar del Empleado, p. 113-146. 